\title{
Evaluación del uso de fibras lignocelulósicas para el reforzamiento de espumas rígidas de poliuretano
}

\section{Evaluation of the use of lignocellulosic fibers for the reinforcement of rigid polyurethane foams}

\author{
DOI: http://doi.org/10.17981/ingecuc.16.1.2020.08
}

Artículo de Investigación Científica. Fecha de Recepción: 09/11/2018. Fecha de Aceptación: 24/09/2019

\author{
Martha Lucía Malagón Micán \\ Universidad de América. Bogotá, D. C. (Colombia) \\ iqmalu@gmail.com \\ Lina María Velásquez Baracaldo \\ Universidad de América. Bogotá, D. C. (Colombia) \\ limayovb@gmail.com \\ William Ferney García Camacho \\ Universidad de América. Bogotá, D. C. (Colombia) \\ garciawilliam159@gmail.com
}

Para citar este artículo:

M. Malagón Micán, L. Velásquez Baracaldo y W. García Camacho, "Evaluación del uso de fibras lignocelulósicas para el reforzamiento de espumas rígidas de poliuretano", INGE CUC, vol. 16, no. 1, 116-127, 2020. DOI: http://doi.org/10.17981/ingecuc.16.1.2020.08

\section{Resumen}

Introducción- Las reservas de petróleo son recursos no renovables, que pueden ser sustituidos por biomasa, proveniente de residuos agroindustriales como las fibras lignocelulósicas, permitiendo de esta manera la sustitución parcial de derivados del petróleo.

Objetivo- Este artículo tiene por objetivo evaluar el uso de fibras lignocelulósicas para el reforzamiento de espumas rígidas de poliuretano.

Metodología- Se realizó un pretratamiento al material lignocelulósico, como cáscaras de mango, cascarilla de arroz y microcristales de celulosa, luego se determinó el contenido de celulosa y lignina, posteriormente se hizo la determinación del contenido del grupo hidroxilo $(\mathrm{OH})$ presente en las fibras y la síntesis de las espumas de poliuretano, incluyendo material lignocelulósico en un 5\%, 10\% y 15\%. Finalmente, se caracterizaron las espumas con la densidad aparente, absorción de agua, resistencia a la tensión y espectroscopia FTIR.

Resultados- La cascarilla de arroz, presentó el mejor resultado con un reemplazo de $\mathrm{OH}$ del 15\%, densidad aparente de $33 \mathrm{~kg} / \mathrm{m} 3$ y resistencia a la tensión $152,92 \mathrm{kPa}$. Para la absorción de agua, las cáscaras de mango alcanzaron un $277 \%$ de incremento en masa, para un $15 \%$ de $\mathrm{OH}$

Conclusiones- El reemplazo de fibras lignocelulósicas, como las cáscaras de mango y la cascarilla de arroz para reforzar espumas rígidas de poliuretano, son una oportunidad para utilizar recursos renovables con mayor valor agregado y aplicaciones industriales. Las espumas reforzadas con cascarilla de arroz al 15\%, son las que tienen mejores resultados y en cuanto a la densidad aparente se obtuvo un valor máximo de $33 \mathrm{~kg} / \mathrm{m}^{3}$, al compararlo con las muestras de cáscara de mango y microcristales de celulosa. Para la resistencia a la tensión, registra un valor de $152,92 \mathrm{kPa}$, con un aumento del $79 \%$ con respecto a la espuma de referencia. Así, estas espumas reforzadas con cascarilla de arroz pueden usarse como material con resistencia a esfuerzos mecánicos y se recomienda evaluar su uso como aislante térmico. De las fibras evaluadas, la cáscara de mango es la mejor opción para una espuma de uso hortícola, ya que obtuvo una capacidad de absorción de agua de $277,30 \%$ correspondiente a la muestra con un porcentaje de sustitución del 15\%

Palabras clave- Espuma rígida de poliuretano; biocompuesto; lignocelulosa; cascarilla de arroz: cáscara de mango; microcristales de celulosa

\section{Abstract}

Introduction- Oil reserves are non-renewable resources, which can be replaced by biomass, from agroindustrial waste such as lignocellulosic fibers, allowing the partial replacement of petroleum derivatives.

Objective- This article aims to evaluate the use of lignocellulosic fibers for the reinforcement of rigid polyurethane foams.

Methodology- A pretreatment of the lignocellulosic material, such as mango husks, rice husk and cellulose microcrystals was carried out, then the cellulose and lignin content was determined, and then the content of the hydroxyl group $(\mathrm{OH})$ present in the samples was determined. fibers and the synthesis of polyurethane foams, including lignocellulosic material by $5 \%, 10 \%$ and $15 \%$. Finally, the foams are characterized with bulk density, water absorption, tensile strength and FTIR spectroscopy.

Results- The rice husk presented the best result with an $\mathrm{OH}$ replacement of $15 \%$, density of $33 \mathrm{~kg} / \mathrm{m} 3$ and resistance to tension $152.92 \mathrm{kPa}$. For the absorption of water, the mango peels reached a $277 \%$ increase in mass, for $15 \% \mathrm{OH}$.

Conclusions-The replacement of lignocellulosic fibers, such as mango husks and rice husks to reinforce rigid polyurethane foams, are an opportunity to use renewable resources with greater added value and industrial applications. Foams reinforced with $15 \%$ rice husks are the ones that have the best results and in terms of bulk density a maximum value of $33 \mathrm{~kg} / \mathrm{m} 3$ was obtained, when compared with the samples of mango peel and cellulose microcrystals. For tensile strength, it records a value of $152.92 \mathrm{kPa}$, with an increase of $79 \%$ with respect to the reference foam. Thus, these foams reinforced with rice husk can be used as a material with resistance to mechanical stresses and it is recommended to evaluate their use as a thermal insulator. Of the fibers evaluated, the mango peel is the best option for a foam for horticultural use, since it obtained a water absorption capacity of $277.30 \%$ corresponding to the sample with a substitution percentage of $15 \%$.

Keywords- Rigid polyurethane foam; biocomposite; lignocellulosic; rice husk; mango peel; microcrystalline cellulose 


\section{INTRODUCCIÓN}

Las reservas de petróleo que existen en la actualidad y el alto impacto que tiene la mentalidad de desarrollo sostenible en el mundo, juegan un papel importante en el uso de productos que son derivados del petróleo. Por lo tanto, es necesario acudir al desarrollo de nuevas tecnologías, que tienen como fin reducir la dependencia del petróleo. Se ha estudiado sobre el concepto de Biomasa como alternativa a la Petroquímica [1], y con la biomasa es posible obtener productos como materiales poliméricos híbridos, biocombustibles, entre otros [2]. Las espumas rígidas de poliuretano, como muchos materiales poliméricos, utilizan dentro de su matriz compuestos derivados del petróleo, en su caso el polietilenglicol, que es el más usado para su fabricación. Entonces se crea una oportunidad de utilizar y aprovechar los residuos agroindustriales como sustitutos parciales de compuestos derivados del petróleo.

Los polímeros de poliuretano se encuentran en diferentes productos como espumas flexibles o rígidas, elastómeros, adhesivos, recubrimientos y sellantes. Entre las aplicaciones más comunes del poliuretano están el uso como aislantes para construcción, aislante en neveras y congeladores, muebles, calzado, recubrimientos, adhesivos, piezas automotrices, entre otros. Los poliuretanos pueden ser espumas de baja densidad de $6 \mathrm{~kg} / \mathrm{m}^{3}$ hasta plásticos con densidades de $1220 \mathrm{~kg} / \mathrm{m}^{3}$ [3].

La biomasa lignocelulósica es la más abundante en la naturaleza y está presente en la madera dura, madera suave, residuos agroindustriales y vegetación. Los residuos lignocelulósicos producidos anualmente a nivel mundial exceden los 220 billones de toneladas, equivalentes a 60-80 billones de toneladas de crudo [4]. Dentro de la lignocelulosa se encuentran tres compuestos fundamentales: celulosa, hemicelulosa y lignina [1].

Las fibras lignocelulósicas pueden ser incorporadas en la estructura de polímeros como las espumas de poliuretano, modificando y mejorando ciertas propiedades físico-mecánicas, reduciendo los costos del polímero. Además, se disminuye el impacto ambiental generado al usar compuestos derivados del petróleo [2]. Este tipo de biomasa, por su contenido de celulosa y lignina posee varios grupos hidroxilos, con pares de electrones libres en cada oxígeno, los cuales pueden reaccionar con grupos funcionales como el isocianato [5].

En diversos estudios alrededor del mundo se ha investigado la incorporación de fibras naturales como la cáscara de maní [6], cáscara de coco [7], cascarilla de arroz [8], cáscara de cacao [9], bagazo de caña de azúcar [10]-[11] y derivados de celulosa [12], entre otros, en la síntesis de espumas de poliuretano, encontrando mejores propiedades físico-mecánicas del material, reducción en los costos y la obtención de polímeros biodegradables.

Por lo expuesto anteriormente, este estudio se centró en el aprovechamiento de residuos agroindustriales de origen nacional, específicamente la cáscara de mango; la cual para el año 2016 la producción en Colombia fue de 270.023 toneladas, según el DANE, de las cuales el 15\% está representado en la cáscara; el otro residuo fue la cascarilla de arroz, que según datos registrados por Fedearroz para el mismo año, se produjeron 631.444 toneladas de arroz [20]. Adicionalmente se evalúa el uso de microcristales de celulosa, la cual es posible obtener a partir de residuos lignocelulósicos y ya ha sido incorporada en matrices poliméricas por sus propiedades estructurales.

Aunque este trabajo considera solamente dos residuos agroindustriales, existe una gran diversidad en el país, que pueden ser caracterizados y aprovechados para la obtención de productos de mayor valor agregado como las espumas de poliuretano, lo que abre las puertas a futuras investigaciones.

\section{REVISIÓN LITERARIA}

\section{A. Poliuretanos}

Los poliuretanos son polímeros obtenidos por la reacción exotérmica entre el isocianato y un poliol, en donde hay un aumento de volumen por el calor generado. Su aplicación se determina por la gran variedad de polioles y aditivos que se pueden incorporar al proceso. Además de catalizadores, surfactantes y agentes espumantes, también pueden usarse extensores de cadena, retardantes de llama y colorantes. 
Las propiedades físicas y mecánicas dependen de los poros formados en la espuma y de su grado de reticulación. La porosidad se origina por el gas formado en la reacción del isocianato con el agua, la cual libera $\mathrm{CO}_{2}$, o por la adición de un agente físico que se evapora. El gas ingresa al polímero, formando una espuma, por la acción del tensoactivo a la reacción. Según esto, el poliuretano se puede clasificar en espumas rígidas, si el gas permanece dentro de la matriz polimérica, y flexibles si el gas no está presente.

\section{B. Polioles e isocianatos}

Son alcoholes con más de un grupo terminal hidroxilo, el isocianato puede ser un Isómero del Toluendisocianato (TDI) o del Metilen-Difenil Diisocianato (MDI).

\section{Biopolioles}

La búsqueda de fuentes alternativas y la dependencia de la industria de polímeros hacia la petroquímica, conlleva al desarrollo de polioles de origen vegetal. Entre los más usados están el aceite de soya y el aceite de maní, entre otros. Los aceites vegetales, a excepción del aceite de ricino, no contienen hidroxilos naturales, es por esto que, con diferentes técnicas de modificación química, se introducen los grupos hidroxilos para reaccionar con los isocianatos [13]. Además, se ha investigado sobre el uso de fibras de origen natural como el bagazo de caña, cáscara de piña, cáscara de coco, cáscara de maní, cáscara de arroz y derivados de celulosa como carboximetilcelulosa, para la incorporación en las espumas de poliuretano, debido a que contienen naturalmente grupos hidroxilos por su contenido de celulosa y lignina.

\section{Espumas rígidas}

Una espuma rígida de poliuretano tiene mayor cantidad de celdas cerradas donde están los gases dispersos, razón por la cual, la utilizan generalmente en la construcción y como aislante térmico en sistemas de refrigeración (cuartos fríos y neveras, entre otros). Ahora bien, debido a su alta reticulación proporciona a su estructura un módulo de elasticidad elevado. En cuanto a las propiedades físicas de una espuma rígida de poliuretano, se destacan densidad, conductividad térmica, estabilidad dimensional con la temperatura y resistencia a la compresión [15].

La densidad es una de las propiedades más importantes en las espumas rígidas de poliuretano, ya que de ésta, se derivan otras propiedades como la resistencia a la compresión, resistencia a la tensión, resistencia a la cizalladura y módulo de corte, entre otras; al tener espumas cada vez más densas, se incrementan y se mejoran diferentes propiedades mecánicas [18]. Existen dos métodos de fabricación de espumas, en frío y en caliente. En el primer caso la síntesis es exotérmica, libera calor en la reacción y se emplea para obtener espumas a partir de un molde predeterminado, utilizando una espumadora sencilla encargada de realizar la mezcla. El producto final obtenido en éste método es más costoso, tiene una calidad superior y su vida útil es más prolongada con respecto a las espumas en caliente [14].

\section{E. Material lignocelulósico}

La biomasa lignocelulósica es la más abundante en la naturaleza y está presente en la madera dura, madera suave, residuos agroindustriales y vegetación. Los residuos lignocelulósicos producidos anualmente a nivel mundial exceden los 220 billones de toneladas, equivalentes a 60-80 billones de toneladas de crudo [4]. Dentro de la lignocelulosa se encuentran tres compuestos fundamentales: celulosa, hemicelulosa y lignina [1].

De los materiales lignocelulósicos, celulosa y hemicelulosa, es posible obtener furfural, xilosas, glucosa, derivados de celulosa, pulpa y combustibles, entre otros. A partir de las ligninas se obtiene vainillina, dimetilsulfuro, fenoles, matrices para geles, fertilizantes y se utilizan para la producción de diversos polímeros, entre otros [1]. Las fibras lignocelulósicas pueden ser incorporadas en la estructura de polímeros como las espumas de poliuretano, modificando y mejorando ciertas propiedades físico-mecánicas, reduciendo los costos del polímero. Además, se disminuye el impacto ambiental generado al no usar compuestos derivados del petróleo [16]. 


\section{Metodología}

Se utilizaron Cáscaras de Mango (CM), Mangifera Indica L. de la variedad Keitt, obtenidas en Corabastos (Bogotá, Colombia); la Cascarilla de Arroz (CA) fue suministrada por una arrocera de Palermo (Huila, Colombia); los Microcristales de Celulosa (MCC) fueron proporcionados por DuPont (Colombia) con un tamaño entre 45-105 $\mu \mathrm{m}$, según el certificado entregado por el proveedor.

Para la síntesis de espumas de poliuretano se utilizaron isocianato, poliol, surfactantes, catalizadores y agentes espumantes, los cuales fueron suministrados por Químicos El Alquimista (Bogotá, Colombia), para la obtención de espumas rígidas, en un sistema de dos componentes conformado por el isocianato MDI (Metil Difenil Isocianato) y el poliol (una mezcla de poliol, catalizador y surfactantes). Los demás reactivos para análisis fueron adquiridos en Disolventes y Soluciones Químicas SAS y Produquímica (Bogotá, Colombia).

\section{A. Pretratamiento físico}

Inicialmente las cáscaras de mango fueron sometidas a un proceso de limpieza para ser secadas en una estufa a $105^{\circ} \mathrm{C}$ durante 10 horas. Luego se pesaron $2 \mathrm{Kg}$ de cada uno de los residuos, cáscaras de mango y cascarilla de arroz, para ser triturados en un molino de martillos marca Siemens, con una potencia de 2HP, a 1710 rpm y motor 1LF3; y posteriormente fueron tamizados para obtener partículas de tamaños, 105-125 $\mu \mathrm{m}$ para las cáscaras de mango y 105-149 $\mu \mathrm{m}$ para la cascarilla de arroz, con porcentajes de humedad de 5,28\% y $3,5 \%$ respectivamente. Para mantener los valores de humedad a lo largo de toda la experimentación, fue necesario almacenar las muestras de fibra en bolsas de aluminio con cierre hermético junto con bolsas de gel sílica.

Los tamaños de partícula deben ser pequeños con el fin de garantizar que la reacción ocurra de una manera eficiente, con un tamaño de partícula menor, se aumenta la superficie de contacto. En la revisión bibliográfica realizada se determina, que se obtienen buenos resultados al trabajar con tamaños de partícula entre 100-180 $\mu \mathrm{m}$. Al tener mayor área superficial se mejora la adhesión interfase en la matriz polimérica, lo que permite mayor interacción con los grupos isocianato [11].

\section{B. Determinación del contenido de celulosa y lignina}

La cantidad de lignina fue determinada mediante el método de Van Soest P.J., en el caso de la celulosa se estimó mediante oxidación con $\mathrm{KMnO}_{4}$ /gravimétrico.

\section{Determinación del número de $\mathrm{OH}$}

Para la determinación del contenido del grupo funcional Hidroxilo $(\mathrm{OH})$ presente en las muestras de fibras, la metodología usada está basada en la norma ASTM D 5155-01, método B [21], la cual es tomada como referencia, dado que no existe una norma para estos materiales. Se hace reaccionar la fibra con un exceso de isocianato y se calienta en un erlenmeyer tapado con un condensador en un baño de maría a $90^{\circ} \mathrm{C}$ y se prosigue con la metodología descrita en la norma. Mediante la equivalencia del isocianato y la cantidad que reacciona con la fibra, se determina el número de $\mathrm{OH}$ en $\mathrm{mg}$ de $\mathrm{KOH} / \mathrm{g}$ de fibra. De esta manera se conoce la cantidad disponible de hidroxilos que tienen las fibras y el poliol para reaccionar con el isocianato, en este caso el MDI.

\section{Preparación de las espumas}

El método seleccionado para la formación de las espumas es el de "one-shot" o método de un solo paso, en donde se mezcla inicialmente la muestra de fibra con el MDI (Metil Difenil Diisocianato) en un recipiente de polipropileno y posteriormente, se adiciona el poliol, se mezcla y se permite el crecimiento de la espuma.

El índice del grupo funcional Isocianato (NCO) seleccionado fue de 1:1, es decir 1.1 equivalentes NCO por cada equivalente $\mathrm{OH}$; Se mantuvo fijo el número total de equivalentes $\mathrm{OH}$ en la mezcla Poliol-fibra para todas las muestras preparadas. Se sustituyó el equivalente de 
OH del Poliol en los siguientes porcentajes: $0 \%, 5 \%, 10 \%$ y $15 \%$, para cada tipo de fibra con el objetivo de garantizar que un exceso de fibra en la formulación no provoque una desestabilización de sus propiedades fisicomecánicas.

Se evaluó el comportamiento de las espumas en cuanto a tiempo y volumen de expansión, usando como método de enfriamiento, durante el crecimiento de la espuma, agua con hielo a $2^{\circ} \mathrm{C}$ en una nevera aislada, experimentando un choque térmico con el fin de evitar que la espuma obtenida no se contraiga ni muestre curvaturas, ya que si se presenta descontroladamente la generación de calor durante la reacción de polimerización, la formación acelerada de gas como agente espumante conlleva a una estructura final poco estable de las celdas formadas en la espuma. Por último, al terminar el tiempo de crecimiento las espumas sintetizadas permanecieron 5 minutos más en el medio frío y se dejaron al medio ambiente durante 3 días antes de desmoldar y cortar.

La formulación utilizada se observa en la Tabla 1, con base en $12 \mathrm{~g}$ de Metil Difenil Diisocianato (MDI), como cantidad fija para cada ensayo. CM: Cáscaras de mango, CA: Cascarilla de arroz, MCC: Microcristales de celulosa.

TABla 1. Formulación de LAS ESPUMAS EVAlUadas

\begin{tabular}{|l|l|l|l|l|}
\hline Muestra & \%Reemplazo OH & Poliol $(\mathrm{g})$ & Fibra (g) & Tipo de fibra \\
\hline S0 & $0 \%$ & 14,65 & 0 & - \\
\hline SCM5 & $5 \%$ & 13,92 & 1,19 & CM \\
\hline SCM10 & $10 \%$ & 13,18 & 2,39 & CM \\
\hline SCM15 & $15 \%$ & 12,45 & 3,59 & CM \\
\hline SCA5 & $5 \%$ & 13,92 & 0,88 & CA \\
\hline SCA10 & $10 \%$ & 13,18 & 1,77 & CA \\
\hline SCA15 & $15 \%$ & 12,45 & 2,65 & CA \\
\hline SMCC5 & $5 \%$ & 13,92 & 0,76 & MCC \\
\hline SMCC10 & $10 \%$ & 13,18 & 1,52 & MCC \\
\hline SMCC15 & $15 \%$ & 12,45 & 2,28 & MCC \\
\hline
\end{tabular}

S0: Muestras sin incorporación de fibra, SCM: Muestras Cáscaras de Mango, SCA: Muestras Cascarilla de Arroz, SMCC: Muestras Microcristales de Celulosa.

\section{E. Caracterización de las espumas}

Las espumas se caracterizaron por medio de la densidad aparente, la absorción de agua y la resistencia a la tensión.

1. Densidad aparente. La densidad de las espumas obtenidas se determinó con base en la norma NTC 237, tomando muestras de 4x3x2 cm y se calculó la densidad en $\left(\mathrm{kg} / \mathrm{m}^{3}\right)$, se realizaron dos repeticiones y con el valor promedio se define la densidad a cada una de las muestras [2].

2. Absorción de agua. La metodología se basó en la norma ASTM D570-81, donde se tomaron muestras de $3 \times 2,5 \times 1,5 \mathrm{~cm}$ y posteriormente se sumergieron en agua a $19^{\circ} \mathrm{C}$ durante 3,5 horas, la cantidad de agua absorbida se determina con la diferencia de pesos final e inicial. Se realizaron dos repeticiones y con el valor promedio se define la absorción de agua.

3. Resistencia a la tensión. La prueba de tensión mecánica se llevó a cabo por triplicado, basándose en la norma ASTM D638, para lo cual las espumas fueron cortadas con dimensiones de 15x2x1 cm y con una velocidad de cabezal de $3 \mathrm{~mm} / \mathrm{min}$, usando un equipo universal de ensayos de tensión SHIMADZU UH-50A.

4. Espectroscopia FTIR. Los análisis se llevaron a cabo a todas las muestras que se presentan en la Tabla 1, con un equipo SHIMADZU IRT Racer-100, en la región de 4000 a $200 \mathrm{~cm}-1$. 


\section{RESUltados}

\section{A. Determinación del contenido de celulosa y lignina}

Los resultados obtenidos para las cáscaras de mango y cascarilla de arroz se observan en la Tabla 2.

Tabla 2. Porcentajes de celulosa y lignina Para las muestras de fibra

\begin{tabular}{|l|l|l|}
\hline \multicolumn{1}{|c|}{ Muestra } & \multicolumn{1}{c|}{ \% lignina } & \multicolumn{1}{c|}{ \% celulosa } \\
\hline CM & 3,76 & 11,77 \\
\hline CA & 30,40 & 28,80 \\
\hline
\end{tabular}

CM: Cáscara de mango, CA: Cascarilla de arroz.

Las cáscaras de mango presentan un porcentaje de lignina menor con respecto a lo reportado por [17] con 4,71\% para cáscaras de mango de la misma variedad Keitt, en cuanto a la cantidad de celulosa el resultado obtenido fue de 7,79\% mayor a lo reportado por los autores [17]. La cascarilla de arroz presenta un alto contenido de lignina en comparación con las cáscaras de mango y con respecto a los valores en la literatura, aproximadamente $24,77 \%$ [5] el contenido es más alto; para las cascarillas de arroz el porcentaje de celulosa se encuentra dentro de los valores reportados en la literatura (28-36\%) [5]. Las variaciones de lignina y celulosa en los residuos, pueden deberse al origen y condiciones de cosecha del fruto y del grano.

\section{B. Determinación del número de $\mathrm{OH}$}

Determinar el contenido de grupos funcionales hidroxilo en las muestras de fibra, permitirá conocer la capacidad de las fibras para reaccionar con los grupos funcionales Isocianato (NCO) presente en el MDI, el valor se reporta en mg de $\mathrm{KOH}$ por cada gramo de fibra. Los valores encontrados para todas las muestras de fibra se presentan en la Tabla 3, se hicieron dos repeticiones bajo condiciones ambientales similares. El resultado es aproximado ya que la humedad en las fibras y en el medio ambiente puede alterar el valor del número de $\mathrm{OH}$.

También se determinó el porcentaje de NCO del MDI encontrando un valor de 24,35 + 0,2. Para el poliol, el certificado del proveedor especifica que el número de $\mathrm{OH}$ es de $240 \mathrm{mg}$ $\mathrm{KOH} / \mathrm{g}$ poliol.

Tabla 3. Número de OH para cada una de las muestras.

\begin{tabular}{|l|l|}
\hline \multicolumn{1}{|c|}{ Muestra } & \multicolumn{1}{c|}{$\mathrm{N}^{\circ} \mathrm{OH}(\mathrm{mg} \mathrm{KOH} / \mathrm{g}$ muestra $)$} \\
\hline Cáscara de mango & $146,65 \pm 2,5$ \\
\hline Cascarilla de arroz & $198,62 \pm 2$ \\
\hline Microcristales de celulosa & $231,26 \pm 1,5$ \\
\hline Poliol & $240,00 \pm 2$ \\
\hline
\end{tabular}

El número de $\mathrm{OH}$ que presentan los polioles usados en la síntesis de espumas de poliuretanos está entre 200 y $800 \mathrm{mg} \mathrm{KOH/g} \mathrm{[18],} \mathrm{esto} \mathrm{indica} \mathrm{que} \mathrm{la} \mathrm{única} \mathrm{muestra} \mathrm{de} \mathrm{fibra} \mathrm{que} \mathrm{se}$ encuentra significativamente por debajo de ese valor es el mango, debido a que contiene menos celulosa y lignina en su composición a comparación con la cascarilla de arroz; en el caso de los microcristales de celulosa el valor es mayor debido a su alto grado de concentración de celulosa con estructura cristalina.

\section{Morfología de las espumas}

Luego de desmoldar y cortar las espumas se observa el tamaño y la homogeneidad de las celdas. Las espumas con incorporación de CM, como se observa en la Fig. 1, presentan una 
estructura casi uniforme y una buena dispersión de las fibras; se pueden observar en las muestras de $10 \%$ y $15 \%$ algunos espacios de aire que quedaron atrapados durante las etapas de mezclado y crecimiento. Adicionalmente, se observa que, a mayor concentración de cáscara de mango, el color de las espumas es más café caramelo.

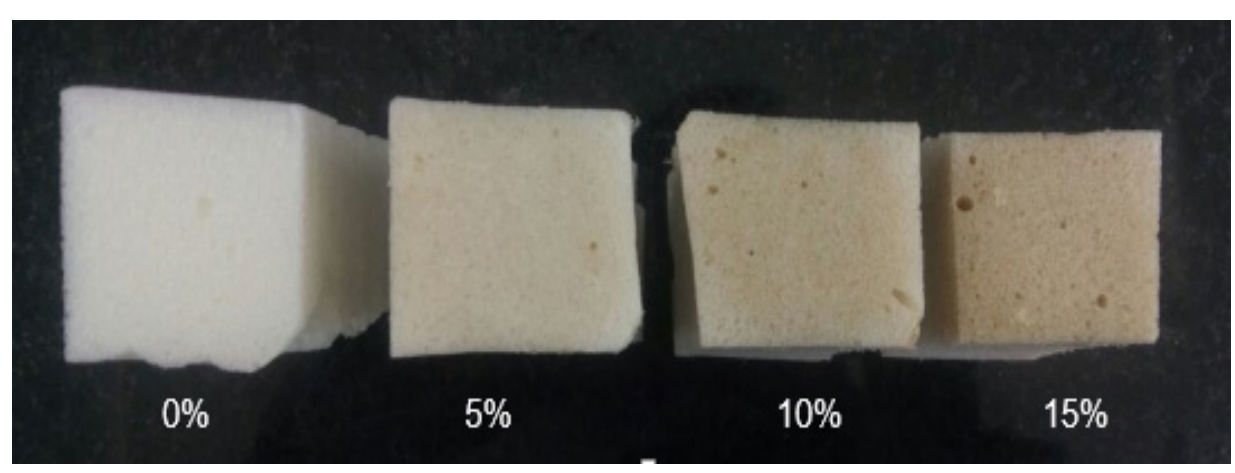

Fig. 1. Estructura de las espumas para los ensayos con cáscara de mango. Fuente: Autores.

Las muestras con CA, como se observa en la Fig. 2, presentan una estructura uniforme y una mejor dispersión de las fibras con respecto a las muestras con CM, se observan espacios de aire únicamente en la muestra de $5 \%$; las muestras con CA se perciben más densas y rígidas que las espumas con CM. Además, se observa coloración a mayor concentración de CA, siendo mucho más intensa la coloración en las espumas obtenidas con mango, debido al incremento inminente de carotenoides en las espumas.

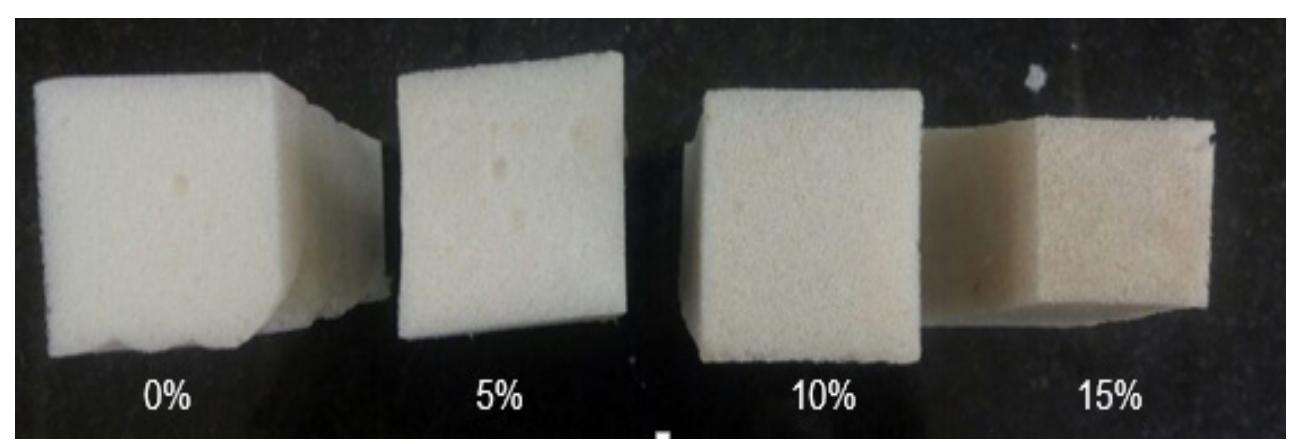

Fig. 2. Estructuras de las espumas para los ensayos con cascarilla de arroz. Fuente: Autores.

Los MCC se dispersan completamente en la mezcla con el MDI y el poliol. El tamaño de poro es uniforme, el cual se determina cualitativamente por observación y solo se evidencia un espacio de aire en la muestra de 15\%; la incorporación de los MCC en la espuma no genera ningún cambio de color significativo, a causa de su coloración, particularmente blanca, ver Fig. 3.

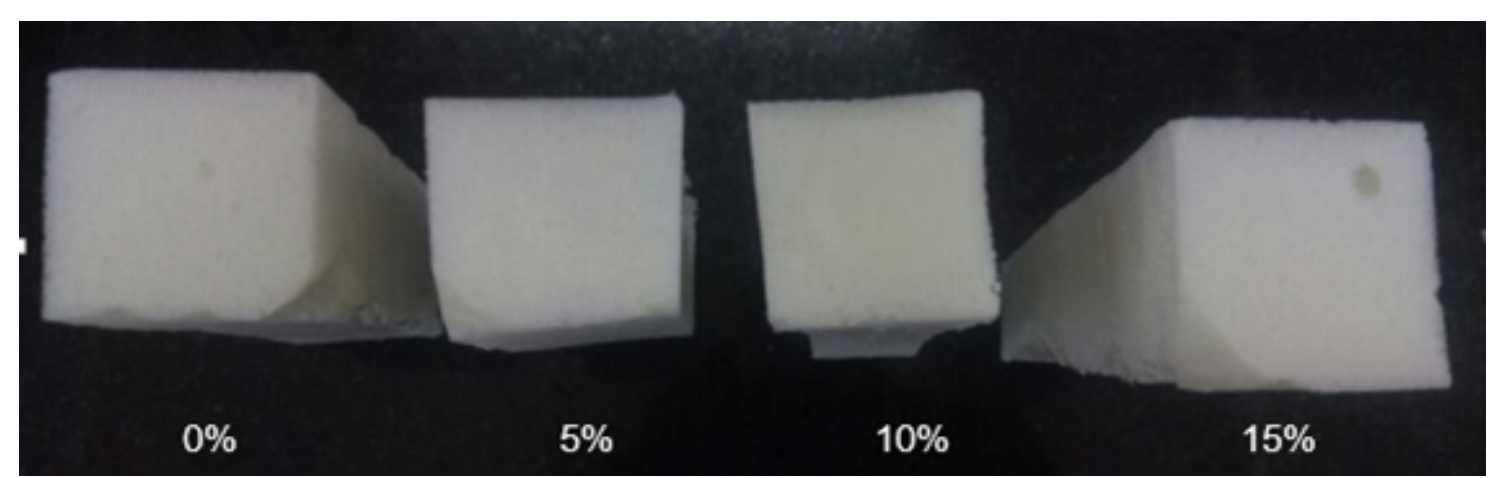

Fig. 3. Estructura de las espumas para los ensayos con microcristales de celulosa. Fuente: Autores.

En general, todas las muestras de espumas rígidas preparadas presentaron un aparente tamaño de poro uniforme, desde un punto de vista cualitativo y se observa un menor volumen de crecimiento final al incrementar el porcentaje de fibra en la formulación.

\section{Densidad aparente}

Los valores que se calcularon para la densidad aparente de las muestras de espuma se representan en la Fig. 4. 


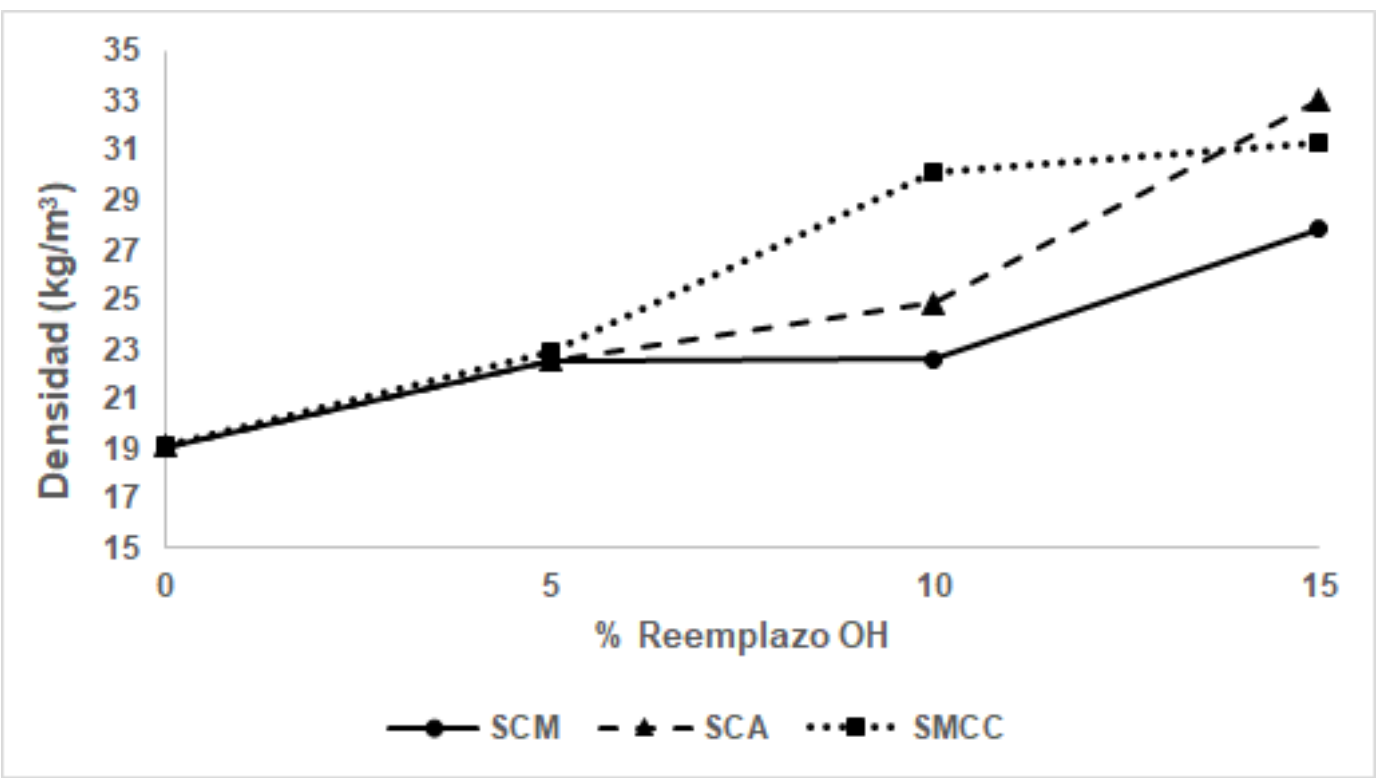

Fig. 4. Efecto del porcentaje de reemplazo $\mathrm{OH}$ en la densidad.

SCM: Muestras Cáscara de Mango, SCA: Muestras Cascarilla de Arroz, SMCC: Muestras Microcristales de Celulosa.

Fuente: Autores.

Las muestras con un porcentaje de reemplazo del $5 \%$ para cualquiera de los tres tipos de fibra poseen una densidad alrededor de $22,5 \mathrm{~kg} / \mathrm{m}^{3}$. Cuando se pasa a un reemplazo del $10 \%$ la muestra con MCC posee la densidad más alta alcanzando $30,11 \mathrm{~kg} / \mathrm{m}^{3}$, mientras que las muestras de CM y CA están por debajo de $25 \mathrm{~kg} / \mathrm{m}^{3}$ siendo la de CM la más baja. Cuando las muestras poseen $15 \%$ de reemplazo de $\mathrm{OH}$, la muestra con $\mathrm{CA}$ alcanza la mayor densidad tomando un valor de $33,04 \mathrm{~kg} / \mathrm{m}^{3}$. Los valores que se encuentran establecidos en la literatura para densidades de espumas de poliuretano rígidas están entre: $20 \mathrm{~kg} / \mathrm{m}^{3}$ a $50 \mathrm{~kg} / \mathrm{m}^{3}$. De esta manera, únicamente la muestra que posee $0 \%$ de reemplazo de fibra se encuentra por debajo de este rango y las muestras con fibra de $5 \%$ a $15 \%$ si se encuentran dentro del rango.

Para las espumas de poliuretano, una propiedad importante es la densidad, ya que al cambiar esta, varían las demás propiedades. Por ejemplo, propiedades como la resistencia a la compresión, tensión, flexión, conductividad térmica y absorción de agua, están en función de la densidad. Por lo general, en las espumas rígidas de poliuretano, las propiedades mecánicas como resistencia a la compresión, módulos de tensión y esfuerzo, incrementan al aumentar la densidad [18]. Por lo tanto, se podría esperar que las espumas con mayor incorporación de fibras en su formulación, tengan mayor densidad por la mayor cantidad de celulosa y lignina cuyas estructuras refuerzan la matriz polimérica y mejoran sus propiedades mecánicas, así como lo han reportado diversos estudios al reforzar espumas de poliuretano con fibras lignocelulósicas.

\section{E. Absorción de agua}

Se analizó la capacidad de absorción de agua de las muestras de espuma preparadas, los valores encontrados se reportan en la Fig. 5.

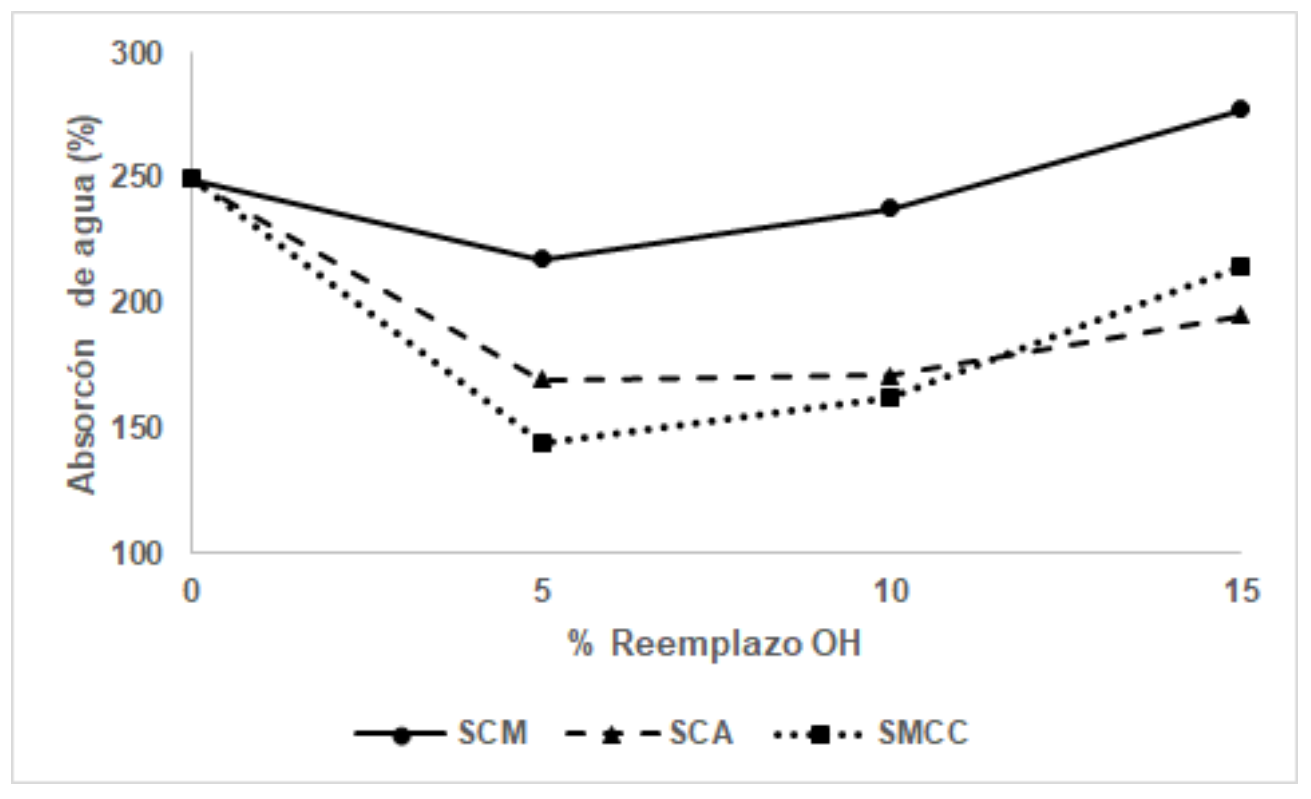

Fig. 5. Efecto del porcentaje de reemplazo $\mathrm{OH}$ en la absorción de agua. Fuente: Autores. 
De acuerdo a lo observado en la Fig. 5 se evidencia que inicialmente, la tendencia de las espumas es disminuir su porcentaje de absorción de agua a medida que aumenta el porcentaje de reemplazo de $\mathrm{OH}$ por parte de las fibras. En el caso de las muestras con $\mathrm{CM}$, se presenta mayor capacidad de absorción de agua con respecto a las otras fibras, siendo a un porcentaje de reemplazo del $15 \%$ un incremento de $277,30 \%$ de masa de la muestra.

Observando la Fig. 4, la densidad aparente aumenta con la concentración de fibras y según la teoría, para una espuma de poliuretano en general, se establece que a mayor densidad disminuye la absorción de agua [18]; por lo tanto, para las muestras de espuma con más de $5 \%$ aprox. la tendencia a disminuir la capacidad de absorción de agua, se ve contrarrestada por la concentración de fibras, ya que este tipo de fibras lignocelulósicas son higroscópicas [19].

\section{F. Resistencia a la tensión}

Las características mecánicas de los materiales son necesarias para determinar la funcionalidad de estos, ante los esfuerzos o cargas que podrían sufrir durante su periodo de uso. Los valores de la resistencia a la tensión de las muestras evaluadas se presentan en la Fig. 6.

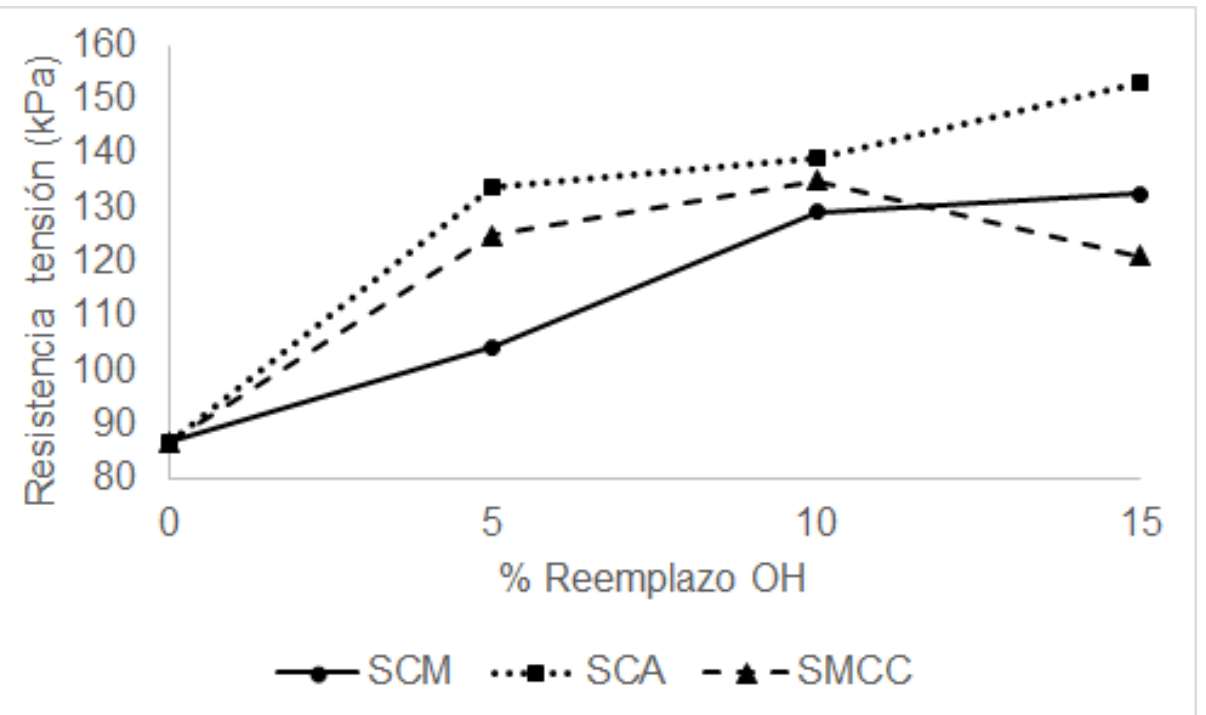

Fig. 6. Efecto del porcentaje de reemplazo de $\mathrm{OH}$ en la resistencia a la tensión. Fuente: Autores.

La Fig. 6 presenta la tendencia de los resultados de las muestras ante el incremento del porcentaje de reemplazo de $\mathrm{OH}$ en la formulación de la espuma; esta tendencia es similar a la que se presenta en densidad aparente y en parte, a la absorción de agua. De los resultados y el análisis obtenido en el efecto de la densidad aparente, se esperaba que a medida que el porcentaje de reemplazo de $\mathrm{OH}$ en las muestras fuera mayor, las propiedades mecánicas se incrementarían y por ende mejorarían. Esto se hace evidente, de forma efectiva en las muestras con incorporación de CM y CA, especialmente, en las muestras con CA que poseen los valores más altos para cada una de las variaciones en el reemplazo, con una resistencia de $152,92 \mathrm{kPa}$ a un porcentaje de reemplazo de $15 \%$.

Las propiedades mecánicas están estrechamente relacionadas con la estructura de la espuma, el tamaño de las celdas y el tipo de celda (abierta, semicerrada y cerrada). Ante la adición de fibras naturales, la estructura de la espuma se vuelve más rígida lo que permite un mayor porcentaje de celdas cerradas, por esto se aprecia el efecto reforzante de las fibras naturales en las espumas de poliuretano, principalmente para las muestras con CA que poseen mayor porcentaje de lignina y celulosa en su composición.

En el caso de las muestras con MCC, al pasar de 10\% al 15\% la resistencia a la tensión disminuye de $135 \mathrm{kPa}$ a $121,16 \mathrm{kPa}$, respectivamente. Se podría afirmar que al 15\% de incorporación, los MCC alcanzan a desestabilizar la estructura; sin embargo, para corroborar dicha afirmación, sería necesario seguir incorporando mayores porcentajes en los MCC y evaluar las propiedades finales de la espuma, verificando que no se puede llevar el porcentaje de reemplazo más allá del $15 \%$.

Con los resultados generales en la mejora de la resistencia a la tensión, puede suponerse que las demás propiedades mecánicas como resistencia a la compresión, módulo de tensión, dureza, entre otras podrían verse también mejoradas con la incorporación de fibras naturales de origen lignocelulósico. 


\section{G. Espectroscopía FTIR}

Los análisis se realizaron a todas las muestras de la Tabla 1. La Fig. 7, Fig. 8 y Fig. 9 muestran un paralelo entre los resultados obtenidos de la muestra referencia con las muestras que tienen porcentajes de sustitución de fibra de 5, 10 y 15\% de cada tipo de fibra.
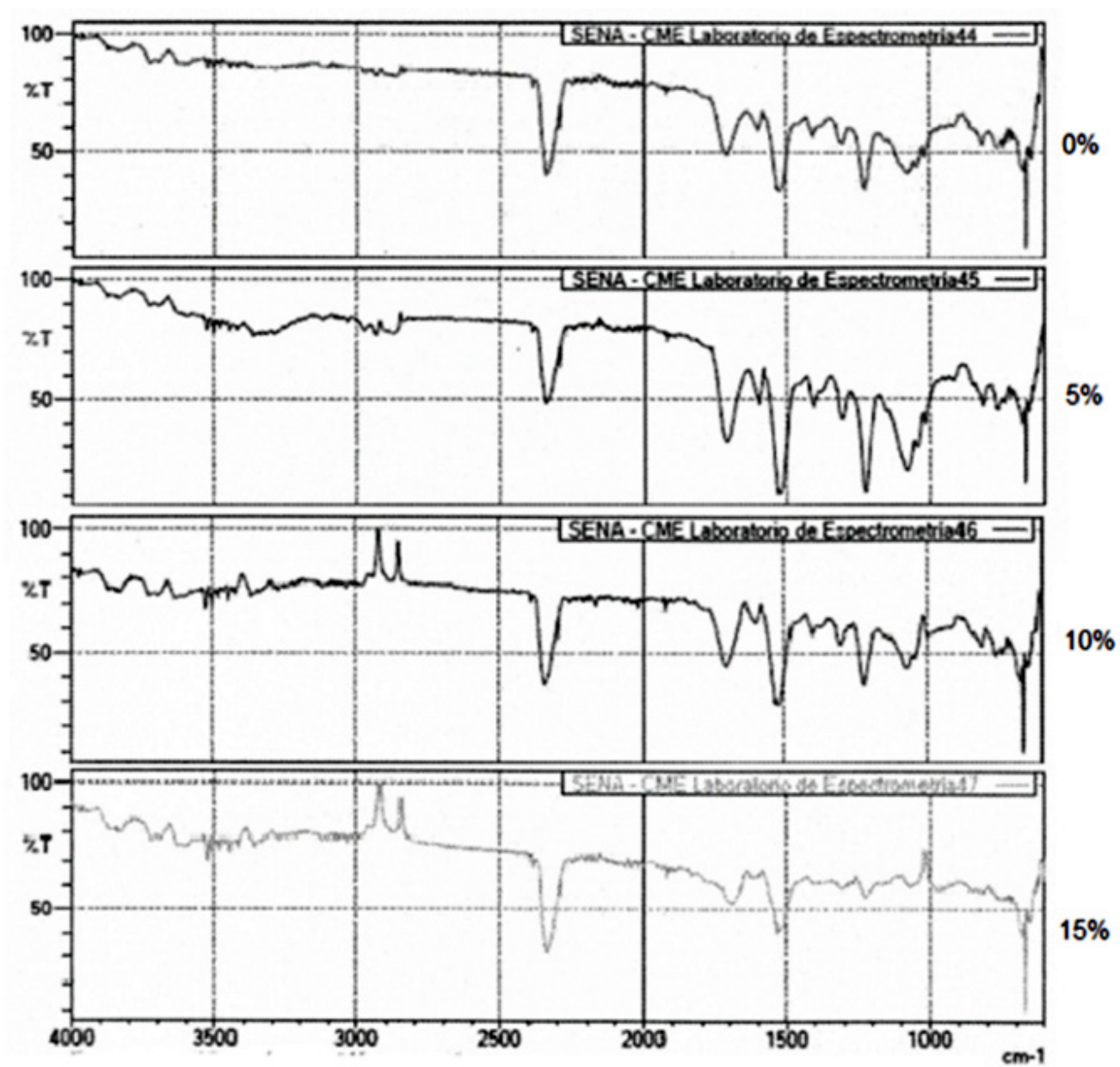

Fig. 7. Espectros infrarrojos para las muestras de espuma con cáscara de mango. Fuente: Autores.
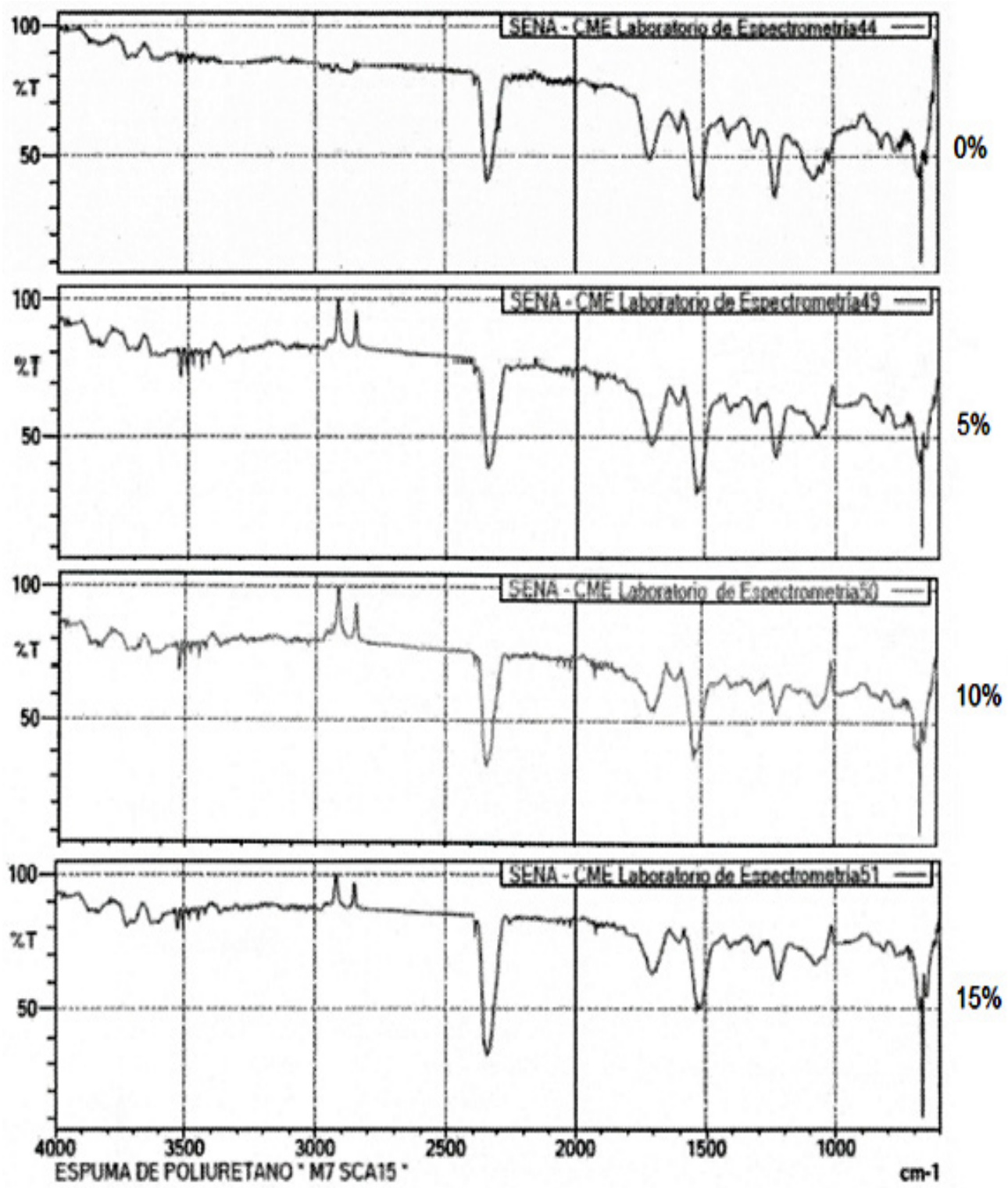

Fig. 8. Espectros infrarrojos para las muestras de espuma con cascarilla de arroz. Fuente: Autores. 
Los espectros observados en la Fig. 7, Fig. 8 y Fig. 9 son los resultados que se obtuvieron a partir de las muestras con CM, CA y MCC respectivamente, teniendo como referencia la espuma que no contiene incorporación de ningún tipo de fibra. En la zona correspondiente a los 2400-2300 $\mathrm{cm}^{-1}$ se pronuncia un pico perteneciente a enlaces $\mathrm{C} \equiv \mathrm{C}$ y C $\equiv \mathrm{N}$; la región comprendida entre los 1700-1600 $\mathrm{cm}^{-1}$ evidencia un pico característico de los grupos carbonilo confirmando la formación de grupos uretano; entre $1600-1500 \mathrm{~cm}^{-1} \mathrm{se}$ forma un pico intermedio generado por la vibración de los enlaces C = C de los anillos del MDI. Entre 1300-1000 $\mathrm{cm}^{-1}$ se presentan unos picos medianos correspondientes a los grupos aromáticos del MDI.
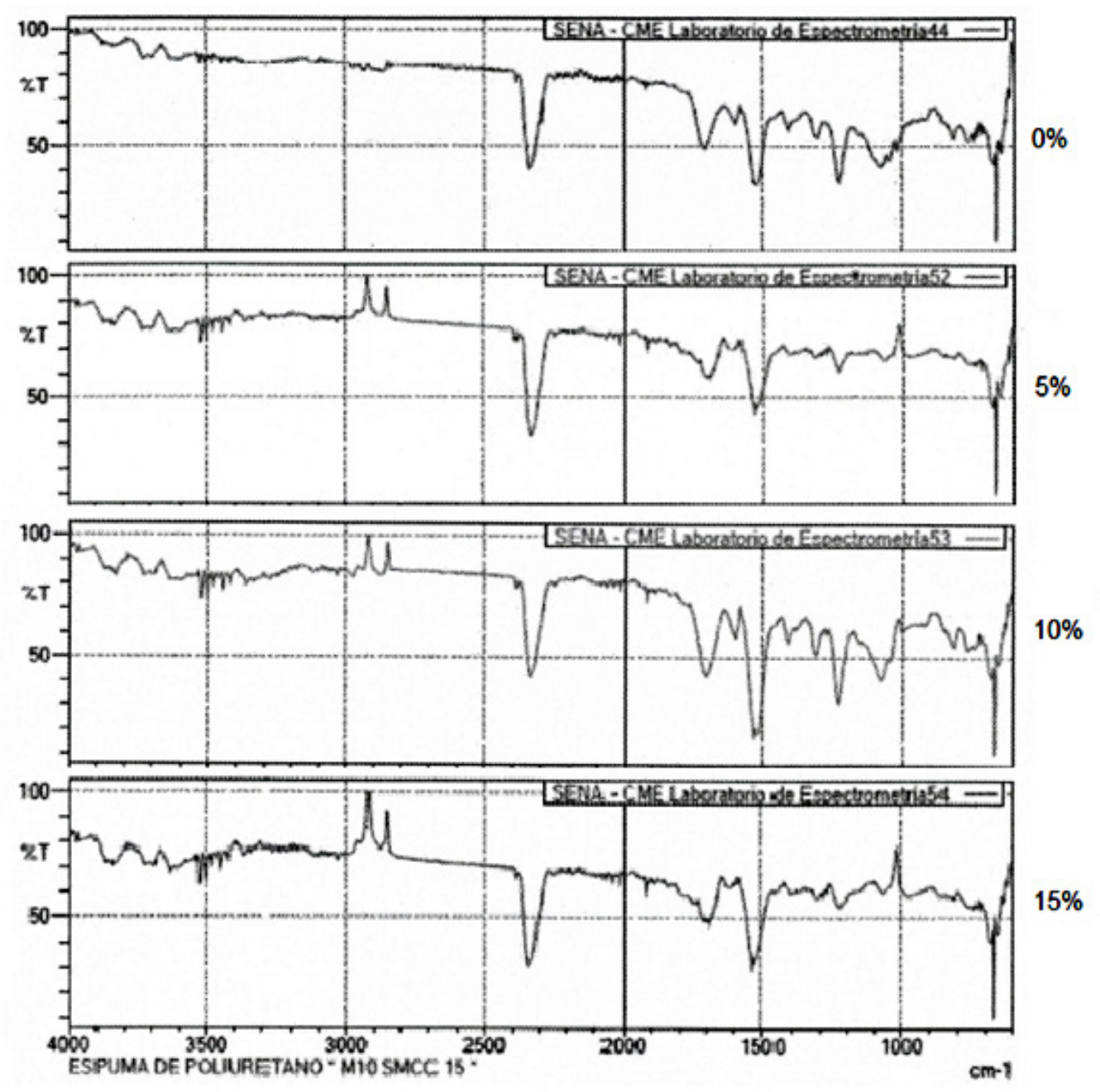

Fig. 9. Espectros infrarrojos para las muestras de espuma con microcristales de celulosa. Fuente: Autores.

En general en todas las muestras se confirma la formación de los grupos uretano y además no se observan los picos característicos de los grupos $\mathrm{OH}$ de la celulosa y lignina de las muestras de fibras, por lo que se podría deducir que todos los grupos $\mathrm{OH}$ libres reaccionaron con los grupos NCO del MDI.

\section{Conclusiones}

El reemplazo de fibras lignocelulósicas, como las cáscaras de mango y la cascarilla de arroz para reforzar espumas rígidas de poliuretano, son una oportunidad para utilizar recursos renovables con mayor valor agregado y aplicaciones industriales.

En el país existe una gran diversidad de residuos agroindustriales, que se pueden aprovechar para la obtención de espumas de poliuretano, con diferentes aplicaciones.

La cascarilla de arroz por su alto contenido de celulosa y lignina, tiene mayor capacidad de reaccionar con los grupos funcionales isocianato del MDI, lo que permite obtener una espuma de poliuretano con mejores propiedades.

Las espumas reforzadas con cascarilla de arroz al $15 \%$, son las que tienen mejores resultados y en cuanto a la densidad aparente se obtuvo un valor máximo de $33 \mathrm{~kg} / \mathrm{m}^{3}$, al compararlo con las muestras de cáscara de mango y microcristales de celulosa. Para la resistencia a la tensión, registra un valor de $152,92 \mathrm{kPa}$, con un aumento del $79 \%$ con respecto a la espuma de referencia. Así, estas espumas reforzadas con cascarilla de arroz pueden usarse como material con resistencia a esfuerzos mecánicos y se recomienda evaluar su uso como aislante térmico. 
De las fibras evaluadas, la cáscara de mango es la mejor opción para una espuma de uso hortícola, ya que obtuvo una capacidad de absorción de agua de $277,30 \%$ correspondiente a la muestra con un porcentaje de sustitución del 15\%; mientras que las espumas con cascarilla de arroz y microcristales de celulosa alcanzaron porcentajes menores.

Aunque, los resultados de este trabajo son satisfactorios, se recomienda realizar más ensayos, con el fin de garantizar su reproducibilidad. Además, medir el tamaño de poro de las espumas por medio de técnicas como la microscopía estereoscópica o por medio de un microscopio SEM para analizar la estructura interna de las mismas.

\section{Financiamiento}

Artículo de investigación científica derivado del proyecto de investigación "Aprovechamiento de materiales lignocelulósicos". Proyecto financiado parcialmente por la Universidad de América en el 2017. Fecha de inicio: febrero de 2017. Fecha de finalización: marzo de 2018.

\section{Agradecimientos}

Los autores expresan sus agradecimientos a la Universidad de América por su apoyo.

\section{REFERENCIAS}

[1] M. Lopretti and A. Gandini, "Nuevos materiales poliméricos derivados de fuentes renovables," INNOTEC, No 7, pp. 59-63, Ene, 2013. Available from https://ojs.latu.org.uy/index.php/INNOTEC/article/ view/200

[2] M. Porras, "Evaluación de la incorporación de celulosa de bagazo de caña en la síntesis de espumas de poliuretano," Tesis grado, Dept. Ing. Qui., UPB, Medellín, CO, 2013.

[3] J. Ocampo, "Criterios de formulación de espumas flexibles de poliuretano MDI basados en la evaluación cualitativa de propiedades finales realizadas a nivel laboratorio," Tesis magistral, Dept. Ing. Qui., UNAL, Bogotá D.C., CO, 2012.

[4] A. Singh, D. Rathore, S. Sevda, I. Abu-Reesh and K. Vanbroekhoven, "Biohydrogen production from lignocellulosic biomass: Technology and Sustainability," Energies, vol 8, no. 11, pp. 13062-13080, Nov. 2015. https://doi.org/10.3390/en81112357

[5] J. Vargas, P. Alvarado, J. Vega-Baudrit y M. Porras-Gómez, "Caracterización del subproducto cascarillas de arroz en búsqueda de posibles aplicaciones como materia prima en procesos," Rev. Cient. Fac. Cienc. Quím. Far., vol 23, no. 1, pp. 86-101, Jan. 2013.

[6] M. Sumalia, B. Ugheoke, L. Timon and T. Oloyede, "A preliminary mechanical characterization of polyurethane filled with ignocellulosic material," LJS, vol. 1, no. 9, pp. 159-166, Dec. 2006. Available from http://ljs.academicdirect.org/A09/get_htm.php?htm=159_166

[7] M. Trujillo, "Desarrollo de un material compuesto de fibras naturales entrecruzadas con poliuretano," Tesis magistral, Universidad de Guadalajara, Jalisco, Guadalajara, MX, 2007.

[8] H. D. Rozman, Y. S. Yeo, G. S. Tay and A. Abubakar, "The mechanical and physical properties of polyurethane composites based on rice husk and polyethylene glycol," Polym. Test., vol. 22, no. 6, pp. 617-623, Sep. 2003. https://doi.org/10.1016/S0142-9418(02)00165-4

[9] G. Padrón-Gamboa, E. M. Arias-Marín, J. Romero-García, A. Benavides-Mendoza, J. Zamora-Rodríguez and S. P. García-Rodríguez, "Efecto de la cáscara de cacao en la obtención de espumas de poliuretano para uso hortícola," Rev. Soc. Quím. Méx, vol. 48, no. 2, pp. 156-164, Abr. 2004.

[10] A. A. Abdel, M. Nassar, A. Emam and M. Sultan, "Preparation and characterization of rigid polyurethane foam prepared from sugar-cane bagasse polyol," Mater Chem Phys, vol. 129, no. 1-2, pp. 301-307, Sep. 2011. https://doi.org/10.1016/j.matchemphys.2011.04.008

[11] G. Huang and P. Wang, "Effects of preparation conditions on properties of rigid polyurethane foam composites based on liquefied bagasse and jute fibre," Polym. Test, vol. 60, pp. 266-273, Jul. 2017. https:// doi.org/10.1016/j.polymertesting.2017.04.006

[12] T. Heinze, J. Rivera-Armenta and A. Mendoza-Martínez, "New polyurethane foams modified with cellulose derivatives," Eur. Polym. J., vol. 40, no. 12, pp. 2803-2812, 2004. https://doi.org/10.1016/j.eurpolymj.2004.07.015

[13] C. Defonseka, Practical Guide to flexible polyuretane foams, Akron, USA: Smithers Rapra Technology, 2013.

[14] K. Ashida, Polyurethane and related foams - Chemistry and Tachnology, Boca Raton, USA: CRC, 2006. https://doi.org/10.1201/9780203505991

[15] M. Quintero, R. Gómez and A. Boyacá, "Los polímeros de poliuretano y la industria colombiana: una oportunidad para el aceite de palma," PALMAS, vol. 28, no. 2, pp. 35-42, Ene, 2007. Available from https://publicaciones.fedepalma.org/index.php/palmas/article/view/1288

[16] A. Proaño, O. Bonilla and M. Aldás, "Desarrollo de un material compuesto de matriz de poliuretano rígido reforzado con fibra de raquis de palma africana," Revista politécnica, vol. 36, no. 2, pp. 1-7, Oct. 2015. Available from https://revistapolitecnica.epn.edu.ec/ojs2/index.php/revista_politecnica2/article/ view/622 
[17] L. Serna and C. Torres, "Potencial agroindustrial de cáscaras de mango (Mangifera indica) variedades Keitt y Tommy Atkins," Acta Agron, vol 64, no 2, pp. 110-115, Abr. 2014. https://doi.org/10.15446/acag. v64n2.43579

[18] M. Szycher, Handbook of polyurethanes, Boca Raton, USA: CRC Press, 2012. https://doi.org/10.1201/ b12343

[19] M. E. Porras, C. P. Muñoz, A. M. Gil and G. C. Quintana, "Obtención de espumas de poliuretano con materiales lignocelulósicos,” Invest. Aplic., vol 6, no. 2, pp. 93-102, Dic. 2012.

[20] W.F. García y L. M. Velásquez, "Evaluación del uso de fibras lignocelulósicas para el reforzamiento de espumas de poliuretano a nivel laboratorio," Proyecto grado, Dept. Ing. Qui., UAmerica, Bogotá D.C., CO, 2018, Disponible en https://repository.uamerica.edu.co/bitstream/20.500.11839/6714/1/61229132018-1-IQ.pdf

Martha Lucía Malagón Micán recibió el título de Magister en Ingeniería Química con énfasis en Bioprocesos en la Universidad Nacional de Colombia y de Magister en docencia en la Univesidad de La Salle (Bogotá, D. C., Colombia). Sus áreas de interés son los Bioprocesos y la producción de alimentos. Actualmente es docente investigador de la Universidad de América. https://orcid.org/0000-0003-1603-9324

Lina María Velásquez Baracaldo recibió el título de Ingeniero Químico en la Universidad de América. Perteneció al grupo de investigación en Bioprocesos BIOTECFUA de la Universidad de América, en el Programa de Ingeniería Química. Sus intereses en investigación incluyen investigación en biocompuestos, el desarrollo de productos y la optimización de procesos. https://orcid.org/0000-0001-6365-7286

William Ferney García Camacho recibió el título de Ingeniero Químico en la Universidad de América. Su investigación está orientada en el aprovechamiento de residuos agroindustriales y tiene como reto obtener productos de alto valor agregado por medio de la biotecnología. Él perteneció al grupo de investigación en Bioprocesos BIOTECFUA de la Universidad de América en el Programa de Ingeniería Química. https://orcid.org/0000-0003-0976-1108 